\title{
Rupture of the sinus of Valsalva during pregnancy
}

\author{
T CRIPPS, C W PUMPHREY, D J PARKER \\ From the Regional Cardiothoracic Unit, St George's Hospital, London
}

SUMMARY A case is described in which rupture of the right sinus of Valsalva occurred at 3 I weeks' gestation. The ruptured sinus was successfully repaired one week after the delivery of $\mathbb{S}^{\circ}$ healthy infant by caesarean section.

Rupture of a sinus of Valsalva has not been reported in pregnancy before. We describe such a case, and outline a successful management strategy.

\section{Case report}

A 22 year old Turkish Cypriot woman in week 37 of her first pregnancy attended her local hospital because of palpitation and chest pain. She had become aware of a sudden increase in her heart rate one week before when hanging out washing: there had been some retrosternal discomfort at the time. Subsequently a dry cough, dyspnoea, and orthopnoea had developed and a heart murmur was heard.

Examination showed tachypnoea on minor exertion. She had a sinus tachycardia of 110 beats $/ \mathrm{min}$. The carotid upstroke was sharp and the cardiac impulse was hyperdynamic. The jugular venous pressure was normal. On auscultation a continuous murmur was most strongly heard in mid-systole and at the left sternal edge. The blood pressure was $130 / 50 \mathrm{~mm} \mathrm{Hg}$ in both arms. Examination was otherwise unremarkable for week 37 of pregnancy.

The chest radiograph showed increased vascular markings and the electrocardiogram was normal. The diagnosis was thought to be rupture of a sinus of Valsalva, though other causes of aortic run-off including aortic dissection with aortic valve regurgitation were considered. Caesarean section was performed and polyhydramnios was found. The patient was delivered of a healthy female infant. Echocardiography showed a probable defect in the right sinus of Valsalva (fig 1) but in view of the possibility of aortic dissection it was felt that angiographic confirmation of the diagnosis should be obtained. Aortography demonstrated the presence of a com-

Requests for reprints to Dr T Cripps, Cardiac Department, St George's Hospital, Blackshaw Road, London SW 17 OQT. munication between the aorta and the right ventricle through the sinus of Valsalva (fig 2).

One week later the patient continued to have evio dence of an important left to right shunt with a rest ing tachycardia and radiographic evidence of pulmonary venous congestion. Repair of the rup tured sinus was undertaken and an aneurysm was found. It arose from the right coronary sinus of Valeo salva and communicated with the right ventricular outflow tract just below the pulmonary valve. ThE defect was repaired with sutures from both the aortis and right ventricular aspects.

\section{Discussion}

Since the first case of rupture of the sinus of Valsalv was described by Thurnam in 1840 over 200 case

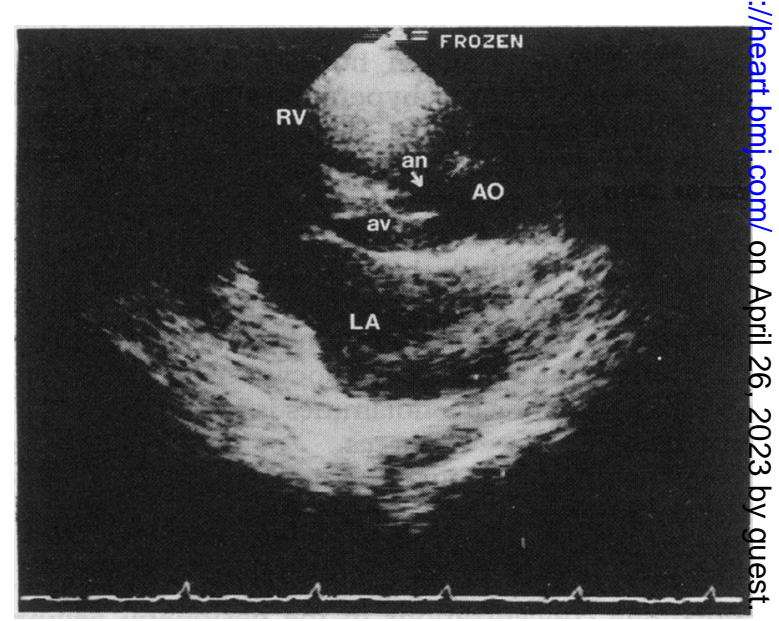

Fig 1 Echocardiogram in long axis parasternal view showing a defect in the anterior aortic wall (an). Flow through the defect was demonstrated with pulsed wave Doppler. $R V$, right ventricle; $L A$, left atrium; $A O$, aorta; av, aortic valve. 


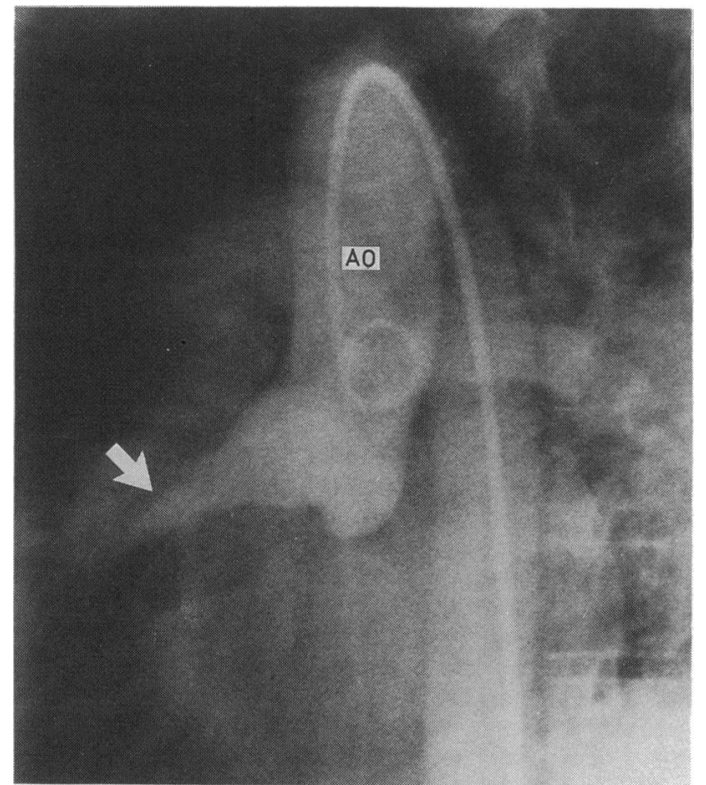

Fig 2 Aortogram in the left anterior oblique projection showing regurgitation of contrast through a ruptured right sinus of Valsalva into the right ventricle. AO, aorta.

have been reported ${ }^{1}$ but this condition has not previously been described in pregnancy. Most cases of aneurysm of the sinus of Valsalva are thought to be congenital, probably resulting from a lack of continuity between the aortic media and the annulus fibrosis. ${ }^{2}$ There may be an associated ventricular septal defect, coarctation of the aorta, or a bicuspid aortic valve. Other cases result from an inherited connective tissue abnormality, as in Marfan's syndrome or Ehlers-Danlos syndrome; from inflammatory disease, as in endocarditis, syphilitic or granulomatous aortitis, or Behçet's disease; and from mechanical disruption after stab wounds, aortic dissection, or after aortic valve replacement or ventricular septal defect repair. A congenital aneurysm may persist into middle age, when it may cause right ventricular outflow obstruction or may rupture into any of the four cardiac chambers, the pulmonary arteries, pericardium, or adjacent extravascular spaces. Echocardiography may allow the detection of the aneurysm before the onset of symptoms. ${ }^{3}$

This case was typical of the congenital type; perhaps rupture was precipitated by the hyperdynamic state of late pregnancy coupled with hormonally induced changes in the mechanical properties of connective tissue. It was felt that since the patient was already haemodynamically compromised, caesarean section was the preferred method of delivery. The risks of allowing vaginal delivery in this condition are unknown, since the condition has not been reported before in pregnancy, but it was thought likely that the haemodynamic changes occurring during labour would exacerbate the left to right shunt and perhaps extend the aneurysmal tear. After delivery, as would be expected, evidence of a continuing important left to right shunt was present, and the defect was corrected. A delay of several days was allowed, to minimise the risk of haemorrhage from the placental site. Although delivery can proceed without complications in heparinised patients, haemorrhage was still a risk. Ergometrine was contraindicated in the presence of a ruptured sinus of Valsalva.

In this case the optimal management was clear because the patient was close to term and so could be delivered without undue risk to the child. Had the patient presented earlier in pregnancy, the decision whether to allow pregnancy to continue would have depended on the balance between the risk to the child of an early delivery and the haemodynamic consequences to the mother of continuing to a later stage of pregnancy. In the event, she did not present until 37 weeks' gestation and the management described resulted in a successful outcome for both mother and child. This report parallels a recent one of acute aortic dissection during pregnancy, ${ }^{4}$ and both illustrate a successful management strategy with prompt caesarean section and definitive early surgery for the maternal condition.

We are grateful to $\mathrm{Mr} M$ Pearce who conducted the obstetric management of this case.

\section{References}

1 Boutefeu JM, Moret PR, Hahn C, Hauf E. Aneurysms of the sinus of Valsalva. Report of seven cases and review of the literature. Am J Med 1978;65:18-24.

2 Edwards JE, Burchell HB. The pathological anatomy of deficiencies between the aortic root and the heart including aortic sinus aneurysms. Thorax 1957; 12:125-39.

3 Desai AG, Sharma S, Kumar A, Hansoti RC, Kalke BR. Echocardiographic diagnosis of unruptured aneurysm of right sinus of Valsalva: an unusual cause of right ventricular outflow obstruction. Am Heart J 1985;109:363-4.

4 Pumphrey CW, Fay T, Weir I. Aortic dissection during pregnancy. $\mathrm{Br}$ Heart $J$ 1986;55:106-8. 